\title{
Resonant Pull-In Condition in Parallel-Plate Electrostatic Actuators
}

\author{
Andreu Fargas-Marques, Jasmina Casals-Terré, and Andrei M. Shkel, Associate Member, IEEE, Member, ASME
}

\begin{abstract}
Electrostatic parallel-plate actuators are a common way of actuating microelectromechanical systems, both statically and dynamically. In the static case, the stable actuation voltages are limited by the static pull-in condition, which indicates that the travel range is approximately limited to $1 / 3$ of the initial actuation gap. Under dynamic actuation conditions, however, the stable voltages are reduced, whereas the travel range can be much extended. This is the case with the dynamic pull-in and the resonant pull-in conditions (RPCs). Using energy analysis, this paper extends the study of pull-in instability to the resonant case and derives the analytical RPC. This condition predicts snapping or pull-in of the structure for a given domain of dc and ac actuation voltages versus quality factor, taking into account the nonlinearities due to large amplitudes of oscillation. Experimental results are presented to validate the analytically derived RPC.

[2006-0147]
\end{abstract}

Index Terms-Electrostatic actuation, nonlinear spring, parallel-plate capacitor, pull-in instability, resonators.

\section{INTRODUCTION}

$\mathbf{M}$ ICROELECTROMECHANICAL systems (MEMS) based on the electrostatic parallel-plate actuation principle are seemingly simple to fabricate and operate while providing relatively large forces (Fig. 1). One challenge is the instability resulting from the nonlinearity of the parallel-plate electrostatic force that occurs at $2 / 3$ of the initial gap, which is known as the static pull-in condition [1].

Several techniques have been considered to increase the stable static range of actuation of parallel-plate actuators. They can be classified in two distinct approaches.

The first approach is based on modifying the design of the electromechanical system, either by introducing mechanical nonlinear leverages [2] or by changing the profile of the electrostatic forces by modifying the design of the capacitor plates [3], [4].

The second approach is based on control strategies, either using position feedback control to prevent snapping, as proposed in [5], or using a series inductance or capacitor in the control circuit [6]. A promising possibility is charge control

Manuscript received July 25, 2006; revised April 17, 2007. Subject Editor G. Fedder.

A. Fargas-Marques is with the Institute of Industrial and Control Engineering, Technical University of Catalonia, 08028 Barcelona, Spain (e-mail: andreu.fargas@upc.edu).

J. Casals-Terré is with the Department of Mechanical Engineering, Technical University of Catalonia, 08222 Terrassa, Spain (e-mail: jasmina.casals@ upc.edu).

A. M. Shkel is with the University of California, Irvine, CA 92697-3975 USA (e-mail: ashkel@uci.edu).

Digital Object Identifier 10.1109/JMEMS.2007.900893

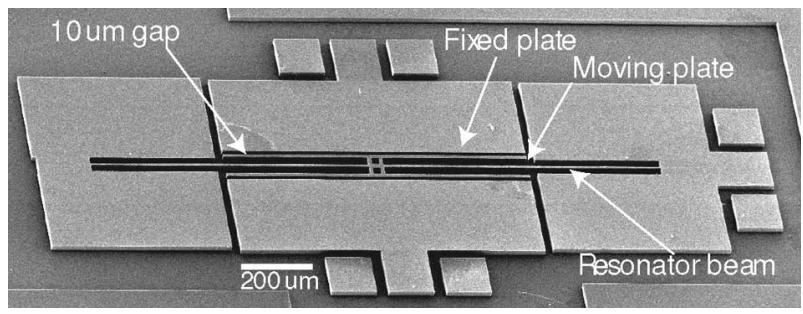

Fig. 1. Beam resonator with parallel-plate electrostatic actuation and sensing. The design parameters are shown in Table I. The device was used to experimentally verify the RPC.

strategies [7], which clearly allow to extend the travel range when compared to voltage-controlled strategies, but they are also limited by the charge pull-in [8].

In most cases, the objective sought is static positioning of the capacitor plates, with dynamics usually ignored or treated as a transient effect. In microrelay applications, where pull-in or gap closing is the objective, dynamics is basically introduced as a factor of time response [9]-[11]. In MEMS positioning studies, dynamics is used as a tool to extend the static travel range [12].

Dynamics play an important role in MEMS devices. Recent studies have explicitly dealt with the implications of dynamics in transient excitation [13] and forced oscillation of MEMS devices [14], [15]. Specifically, parametric excitation has also been addressed [16], [17].

Moreover, in many MEMS applications, the devices are operated at resonant frequencies. This is the case in resonant force sensors [18], accelerometers [19], and gyroscopes [20]. In these applications, large amplitudes of motion are needed to improve sensing capabilities. Consequently, nonlinear spring effects add up to the electrostatic nonlinear forces, leading to reduced stable driving voltages.

The issue of stability limitations in oscillatory excitation was first presented in [21] for double-sided actuated gyroscopes. The study included experimental results. Since then, theoretical and simulation analysis of the nonlinear oscillation behavior and the study of the mechanisms that lead to dynamic pull-in have been presented in [22] for primary-resonance excitation and in [23] for subharmonic and superharmonic excitations. These studies have led to propose using resonant pull-in as a way of reducing voltage excitation in radio frequency MEMS switches [24]. At the same time, a method to increase the dynamic pull-in voltage (DPV) using optimal control has been discussed in [25].

This paper examines the problem of resonant stability and derives for the first time the constructive analytical resonant 


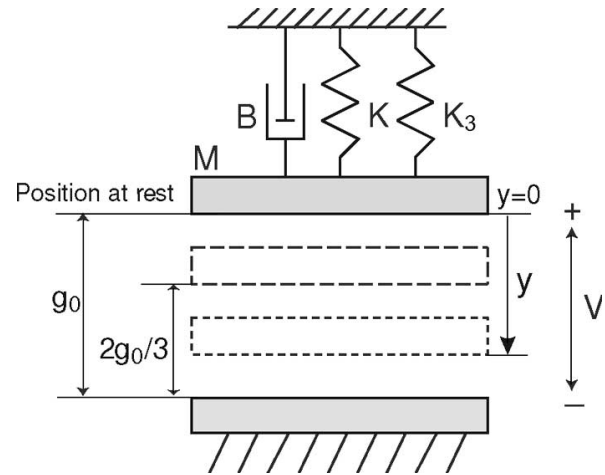

Fig. 2. Schematics of an electromechanical system with parallel-plate actuation. In the linear spring case $\left(K_{3}=0\right)$, the static pull-in occurs when the distance between plates is $2 / 3$ of the initial gap. In the dynamic case, we are studying the maximum achievable amplitude of oscillation $y$ beyond the static snapping condition.

pull-in condition (RPC). The presented analysis takes into account energy storage, transfer, and power dissipation in steady-state response of electrostatically actuated dynamic MEMS structures. The derived stability limits represent upper limits to the dynamic pull-in, beyond which no stable motion can exist. There are also other factors that affect the stability, which are related to transient response or jumps in the evolution of vibratory motion [22]; however, these factors are not the subject of this paper. This paper is an extension of [26] and [27], which were previously published by this group.

In Section II, the model used in [26] is extended to account for the nonlinear spring effect that appears with large amplitudes of oscillation. Using this model, the static, dynamic, and resonant pull-in are analyzed in Section III. Specifically, the analytical expression derived in [28] to calculate the static pull-in as a function of the nonlinear spring is extended to the dynamic pull-in case.

Following this analysis, in Section IV, the RPC is calculated for the nonlinear spring model case. The result of the calculation shows that the RPC derived in [27] is applicable to the case of the nonlinear spring. Finally, new experimental results are presented in Section $\mathrm{V}$ that validate the proposed approach. Results and conclusions are summarized in Section VI.

\section{Nonlinear Actuator Energy Model}

We study the issue of dynamic instability using a concentrated-parameter mass-spring-damper model (Fig. 2), with the commonly used assumption of voltage-controlled actuation [2]-[6]. This is a good first-order representation of a wide variety of MEMS devices, including electrostatically actuated rigid bodies on an elastic beam suspension, as shown in Fig. 1.

To describe the phenomena, we consider the energy exchange that exists in electrostatically driven MEMS beam resonators.

The kinetic energy $\mathbf{T}$ that is associated with a moving mass $M$ is

$$
\mathbf{T}=\frac{1}{2} M \dot{y}^{2}
$$

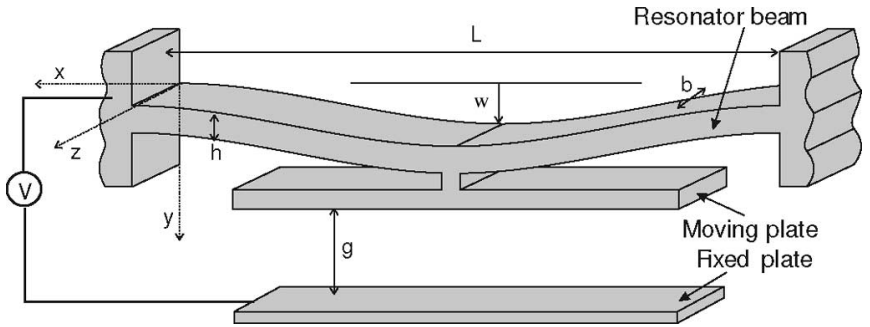

Fig. 3. Basic scheme of a deflected beam with electrostatic parallel-plate actuation as the ones used for testing. Using the Galerkin method, the deformation of the beam is studied as the lumped mass-spring-damper system in Fig. 2.

where $\dot{y}$ is the velocity along the $y$-direction. In a general case of oscillation of elastic beams (Fig. 3), this kinetic energy depends on the oscillation amplitude $w(x, t)$, i.e.,

$$
\mathbf{T}=\frac{\rho b h}{2} \int_{0}^{L}\left(\frac{\partial w}{\partial t}\right)^{2} d x
$$

where $\rho$ is the density of the beam, $h$ and $b$ are the width and thickness of the beam, respectively, and $L$ is the length of the beam.

To obtain the concentrated-parameter mass constant $M$ from the distributed-parameter formulation (2), the Galerkin method is usually applied [29]. The method assumes that the beam response is composed of an infinite number of oscillation modes $w(x, t)=\sum_{i} q_{i}(t) \phi_{i}(x)$, where $q_{i}(t)$ is the time-dependent modal displacement for the oscillation mode $i$, and $\phi_{i}(x)$ is the position-dependent modal shape. Using this approach, the equivalent mass for each mode of oscillation $i$ can be obtained [29], i.e.,

$$
M_{\mathrm{eq}, i}=\rho b h \int_{0}^{L} \phi_{i}^{2} d x .
$$

If a parallel plate is attached to the center of the beam, as in the case of Fig. 3, the equivalent mass has to be corrected with the mass of the driving plate [29] to capture all dynamic effects, and the corrected equivalent mass is

$$
M_{\mathrm{eq}, i}=\rho b h \int_{0}^{L} \phi_{i}^{2} d x+m_{p} \phi_{i}\left(x_{p}\right)^{2}
$$

where $m_{p}$ is the plate mass, and $x_{p}$ is the position of the geometric center.

The potential energy stored in a deformed suspension (or spring) can be defined, in concentrated parameters, as

$$
\mathbf{U}_{k}=\frac{1}{2} K y^{2}+\frac{1}{4} K_{3} y^{4}
$$

where $K$ is the linear spring constant of the suspension, and $K_{3}$ is the coefficient corresponding to the nonlinear cubic component of the nonlinear spring.

In the case of deformation of a beam suspension (Fig. 3), the associated potential energy has three main components. 
The potential energy generated due to deformation of the beam is proportional to its curvature $\partial^{2} w / \partial x^{2}$, i.e.,

$$
\mathbf{U}_{\mathrm{def}}=\frac{E I}{2} \int_{0}^{L}\left(\frac{\partial^{2} w}{\partial x^{2}}\right)^{2} d x
$$

where $E$ is the Young's modulus, and $I$ is the moment of inertia of the beam cross section. The energy term that comprises the deformation generated by external axial forces $N$ on the beam (e.g., residual stresses) is

$$
\mathbf{U}_{N}=\frac{N}{2} \int_{0}^{L}\left(\frac{\partial w}{\partial x}\right)^{2} d x
$$

which is proportional to the axial force. Finally, the energy of the deformation due to self-stretching is

$$
\mathbf{U}_{\mathrm{int}}=\frac{b h E}{8 L}\left[\int_{0}^{L}\left(\frac{\partial w}{\partial x}\right)^{2} d x\right]^{2}
$$

which appears in the case of large oscillation amplitude [14]. Consequently, the total potential energy associated with the beam is

$$
\mathbf{U}_{k}=\mathbf{U}_{\text {def }}+\mathbf{U}_{N}+\mathbf{U}_{\text {int }} \cdot
$$

Again, the Galerkin method can be used to calculate the concentrated-parameter equivalent spring constants $K$ and $K_{3}$ for each oscillation mode $i$. Thus

$$
\begin{aligned}
K_{\mathrm{eq}, i} & =E I \int_{0}^{L}\left(\frac{\partial^{2} \phi_{i}}{\partial x^{2}}\right)^{2} d x+N \int_{0}^{L}\left(\frac{\partial \phi_{i}}{\partial x}\right)^{2} d x \\
K_{3, \mathrm{eq}, i} & =\frac{b h E}{2 L}\left[\int_{0}^{L}\left(\frac{\partial \phi_{i}}{\partial x}\right)^{2} d x\right]^{2} .
\end{aligned}
$$

These expressions indicate that in the case of large amplitude of oscillation, the beam behaves not only as a linear spring but also as a nonlinear cubic spring [2].

Finally, the electrostatic potential energy associated with the actuator capacitor is

$$
\mathbf{U}_{e}=-\frac{1}{2} \frac{C_{0}}{\left(1-\frac{y}{g_{0}}\right)} V^{2}
$$

where

$$
C_{0}=\frac{\varepsilon_{0} A_{c}}{g_{0}}\left(1+0.65 \frac{g_{0}}{b}\right)
$$

is the capacitance at rest using a first-order fringing field correction [30], $\varepsilon_{0}$ is the dielectric constant, $g_{0}$ is the initial gap between the plates, $b$ is the device thickness, $A_{c}$ is the area of the plates, and $V$ is the applied voltage between the electrodes.
Using the previous definitions, the energy of the whole system $\mathbf{E}$ can be defined as

$$
\mathbf{E}=\mathbf{T}+\mathbf{U}_{k}+\mathbf{U}_{e}
$$

Assuming, as usual, that the system behavior is sufficiently captured by the first mode of oscillation, the dynamic response of the beam in Fig. 3 can be modeled by the lumped mass-spring-damper in Fig. 2, given that $q_{1}(t) \simeq y(t)$, $M_{\mathrm{eq}, 1} \simeq M, K_{\mathrm{eq}, 1} \simeq K$, and $K_{3, \mathrm{eq}, i} \simeq K_{3}$. The consideration of higher order modes would improve the accuracy of the model, as shown in [31], but at the expense of the complexity of the mathematical tools to be used.

Consequently, the dynamics of the system are derived using Lagrange's formulation, introducing the damping force [30] $F_{d}=-B \dot{y}$ as the only nonconservative force contributing to the work $\mathbf{W}$ of the system, i.e.,

$$
M \ddot{y}+K y+K_{3} y^{3}-\frac{1}{2} \frac{C_{0}}{g_{0}\left(1-\frac{y}{g_{0}}\right)^{2}} V^{2}=-B \dot{y} .
$$

This is the dynamics equation of a concentrated-parameter mass-spring-damper with parallel-plate electrostatic actuation and a nonlinear spring.

\section{Pull-In ANALYSis}

Consider the lumped model (14) derived in the previous section. With the usual assumption of voltage-controlled actuation, the pull-in instability is the main limitation to the position of the capacitor plates in the gap.

The analysis of the evolution of the energy of the system (13) is used to determine the equilibrium positions of the system, as well as the regions of instability. Dynamics are studied as an important factor affecting the stability of the system.

\section{A. Static Case}

In static equilibrium $\ddot{y}=\dot{y}=0$, the energy of the system (13) consists only of potential energy terms. Thus

$$
\mathbf{E}=\frac{1}{2} K y^{2}+\frac{1}{4} K_{3} y^{4}-\frac{1}{2} \frac{C_{0}}{\left(1-\frac{y}{g_{0}}\right)} V^{2} .
$$

As a result, the distribution of the system energy along the gap between the electrodes is constant and unique for each voltage applied, as can be observed in Fig. 4.

For low voltages, the energy profile is composed of a stable equilibrium position near the initial position of the system and an unstable equilibrium position near the opposing capacitor plate (e.g., 10-50 V in Fig. 4). As the voltage increases, both equilibrium positions migrate until they merge into an inflection point of the energy curve (e.g., $91.69 \mathrm{~V}$ in Fig. 4). Once this voltage limit is reached, no equilibrium positions exist. The limiting condition for the existence of a stable equilibrium is the presence of an inflection point in (13) defined by $d^{2} \mathbf{E} / d y^{2}=0$. This condition provides the analytical value for the maximum static stable displacement from the initial equilibrium $\left(y_{\mathrm{SPV}}\right)$ and the voltage needed to reach this position. This voltage limit 


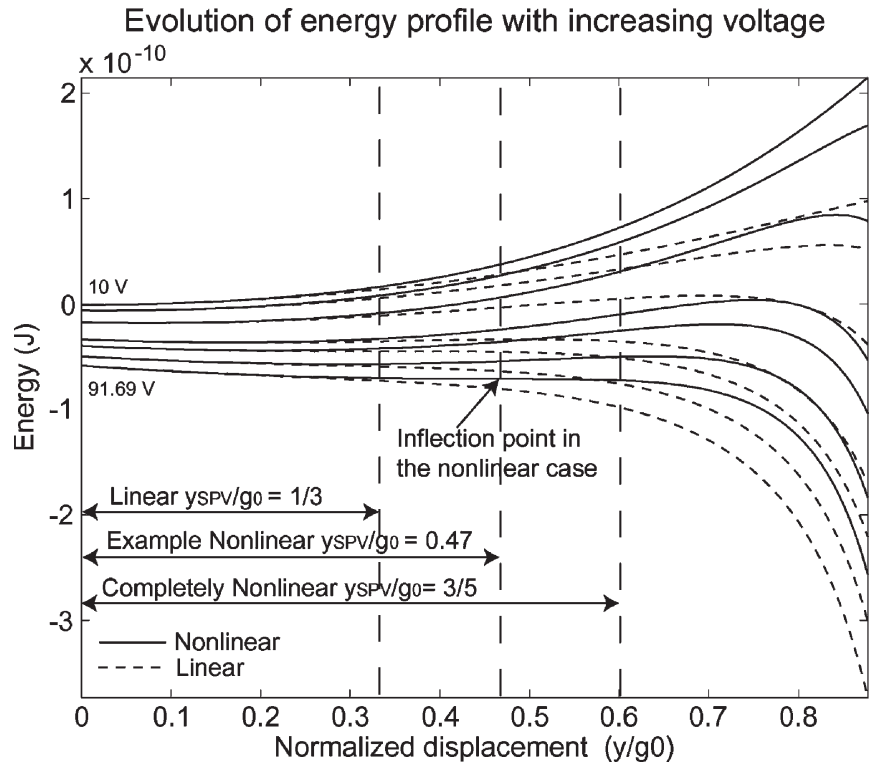

Fig. 4. Potential energy of the system versus normalized displacement for different applied voltages are displayed $(10,30,50,69.45,75.61,84.41$, and $91.69 \mathrm{~V})$, including the SPV and the DPV of the example for the linear case $(75.61$ and $69.45 \mathrm{~V})$ and nonlinear case (91.69 and $84.41 \mathrm{~V})$. The difference between the energy profiles of the linear and nonlinear cases for the same applied voltage shows the importance of the nonlinear spring effect as the actuator moves far away from the rest position.

is the static pull-in voltage (SPV). The values can be obtained analytically using the following formulas [28]:

$$
\begin{aligned}
y_{\mathrm{SPV}}= & \frac{g_{0}}{5}+g_{0}\left[\frac{5 \beta+1}{125}+\frac{\sqrt{5 \beta^{3}-2 \beta^{2}+\beta}}{25}\right]^{\frac{1}{3}} \\
& -g_{0} \frac{5 \beta-1}{25}\left[\frac{5 \beta+1}{125}+\frac{\sqrt{5 \beta^{3}-2 \beta^{2}+\beta}}{25}\right]^{-\frac{1}{3}} \\
\mathrm{SPV}= & \sqrt{\frac{2 K g_{0}^{2}}{C_{0}} \frac{y_{\mathrm{SPV}}}{g_{0}}\left(1+\frac{y_{\mathrm{SPV}}^{2}}{\beta g_{0}^{2}}\right)\left(1-\frac{y_{\mathrm{SPV}}}{g_{0}}\right)^{2}} .
\end{aligned}
$$

In these expressions, $\beta=K /\left(K_{3} g_{0}^{2}\right)$. The importance of the introduction of the nonlinear spring $K_{3}$ can be observed in Fig. 4, where the potential energy curves using a linear stiffness model and nonlinear stiffness model are plotted. For small displacements from the rest position, the influence is negligible, but as the displacement is increased, the effect becomes important. In the example, pull-in instability occurs when the moving plate reaches to $47 \%$ of the total gap displacement, which is farther than the usual $1 / 3$ value.

For the case of linear spring assumption $K_{3}=0$, the resulting value is the classical SPV and its corresponding displacement, i.e.,

$$
\mathrm{SPV}=\sqrt{\frac{8}{27} \frac{K g_{0}^{2}}{C_{0}}} \quad y_{\mathrm{SPV}}=\frac{g_{0}}{3}
$$

The study of (16) and (17) reveals that the maximum displacement is obtained when the spring is completely nonlinear $(K=0)$, and this maximum displacement is $3 / 5$ of the initial gap [28]. This can be observed in Fig. 5, where

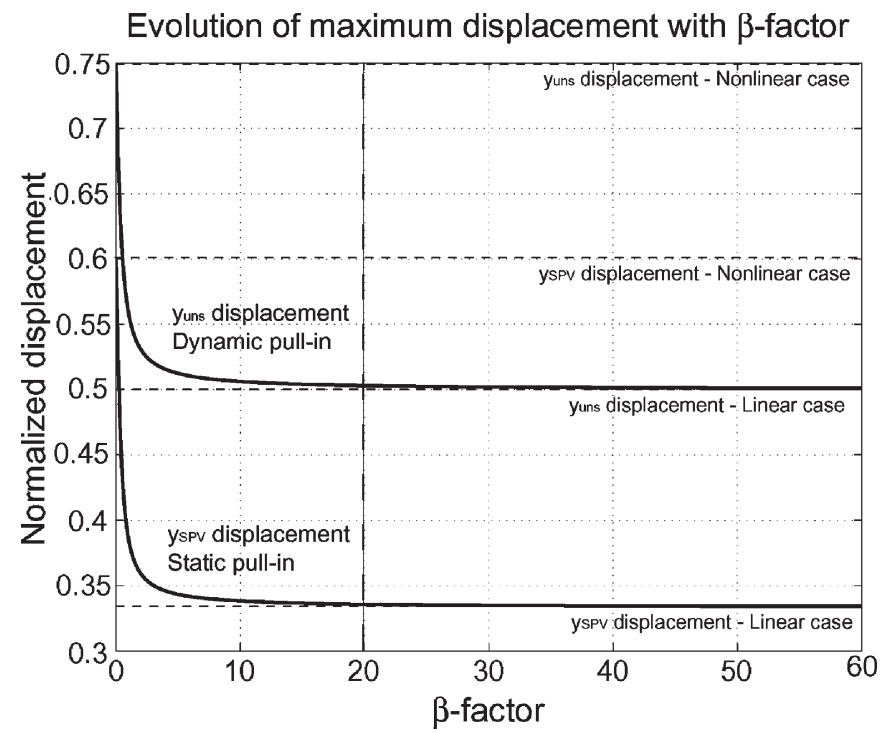

Fig. 5. Influence of $\beta$ factor on the value of the static pull-in displacement and the dynamic pull-in displacement. As can be observed, for values of $\beta$ smaller than 20 , the nonlinear constant begins to influence the result. For values of $\beta$ smaller than 2, the nonlinear constant cannot be neglected.

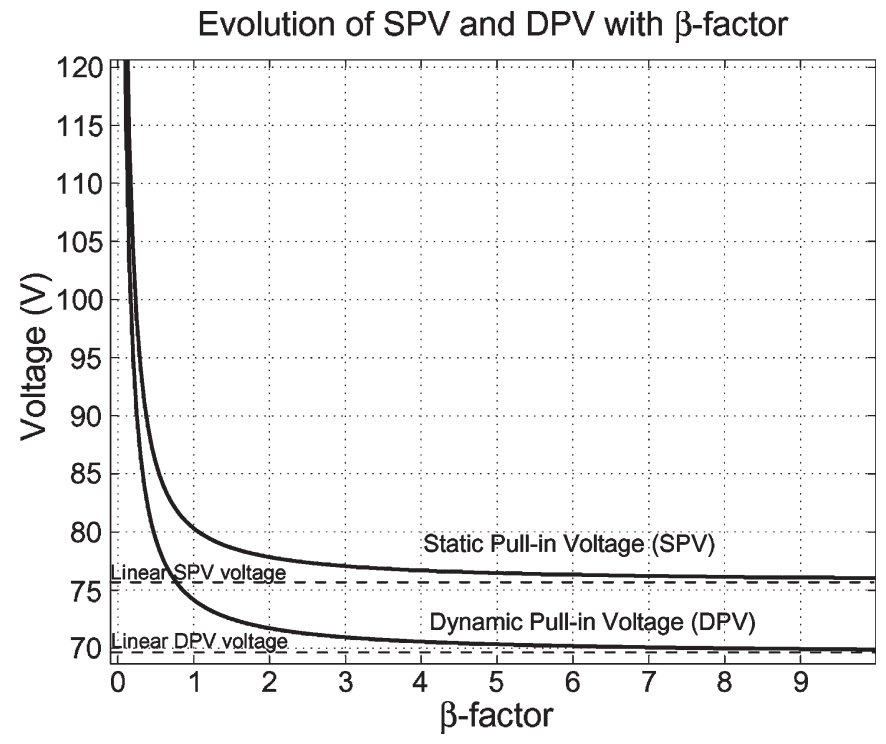

Fig. 6. Influence of $\beta$ factor on the value of the SPV and the DPV for one of the fabricated resonators. To calculate the values, the linear parameters are used, and the nonlinear spring is increased to observe the effect on the pull-in voltages.

it is shown that the nonlinearity is important for values of $\beta$ smaller than 20. The same evolution appears for the SPV values, as shown in Fig. 6. Increasing the nonlinearity in the mechanical spring increases the SPV.

\section{B. Dynamic Case}

The derivations for the static case neglect the transient effects that occur in the system when the voltage is applied. In some cases, such approximation is correct, for example, if the voltage is slowly applied or the system is highly damped. However, for low damping, e.g., in a vacuum environment, the transient dynamics must be taken into account. 


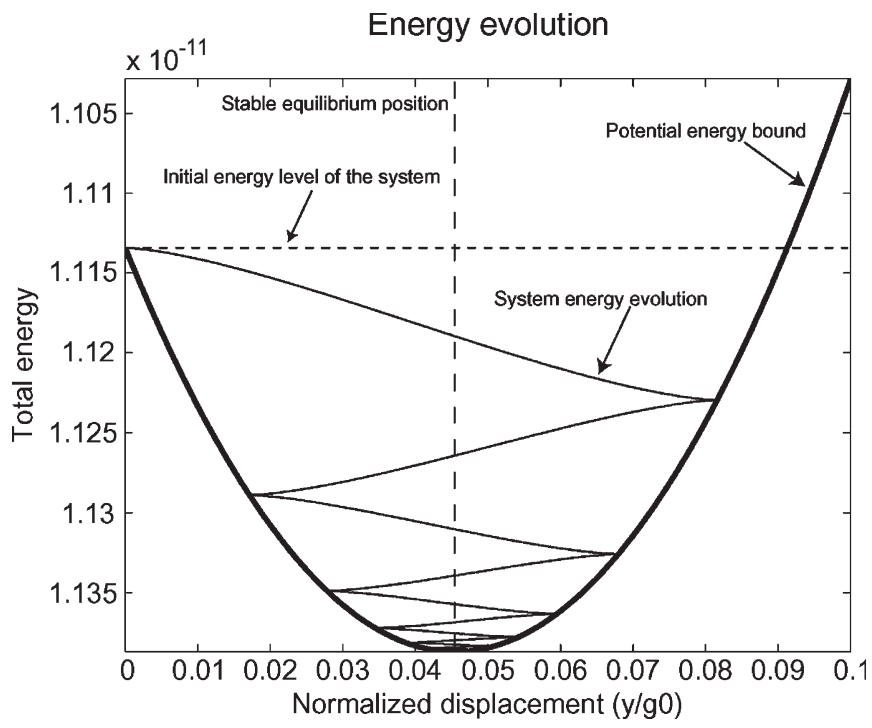

Fig. 7. Evolution of system's energy when a 40-V step function is applied. In the example, the quality factor is 7 . The initial energy corresponds to the potential energy (mechanical and electrostatic). When the motion begins, the potential energy is converted to kinetic energy and dissipation due to damping forces. The system's energy descends until reaching the stable equilibrium position.

The energy analysis can be expanded to account for the transient dynamics of the system when an actuation voltage is applied.

The time derivative of the system energy as defined in (13), $d \mathbf{E} / d t=-B \dot{y}^{2}$, indicates that, unless energy is continuously pumped into the system, the energy decreases with time from its initial energy value until it reaches an equilibrium state, $d \mathbf{E} / d t=0$. According to the model, the only factor that defines the pattern of the energy decay is the damping $B$ of the system.

As can be observed in Fig. 7, the maximum amplitude of displacement of the moving plate is limited by the potential energy bound. If the voltage is increased, at some point, the initial energy of the system and the energy at the unstable peak have the same magnitude (Fig. 4). Assuming that the system has no damping, the total energy of the system remains constant, which implies that when applying a higher voltage, the system will move until it overshoots the unstable equilibrium $y_{\text {uns }}$, and the electrodes will collide. This voltage limit is called the dynamic pull-in voltage (DPV). Any voltage lower than DPV magnitude cannot produce snapping.

To obtain the DPV, the potential energy at rest must be equated to the energy at the unstable equilibrium. This will give the maximum amplitude that can be reached during the step evolution $\left(y_{\text {uns }}\right)$ and the maximum voltage that can be applied (DPV). Using the same terminology as in (16), the displacement expression is

$$
\begin{gathered}
y_{\mathrm{uns}}=\frac{g_{0}}{4}+g_{0}\left[\frac{8 \beta+1}{64}+\frac{\sqrt{768 \beta^{3}-108 \beta^{2}+162 \beta}}{144}\right]^{\frac{1}{3}} \\
-g_{0} \frac{16 \beta-3}{48}\left[\frac{8 \beta+1}{64}+\frac{\sqrt{768 \beta^{3}-108 \beta^{2}+162 \beta}}{144}\right]^{-\frac{1}{3}}
\end{gathered}
$$

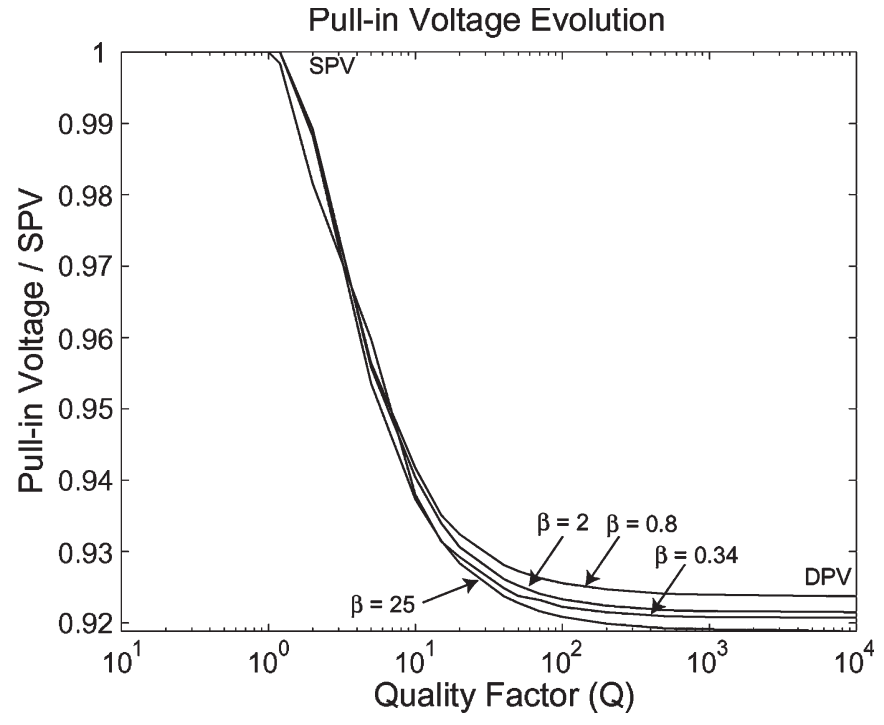

Fig. 8. Comparison of the evolution of the pull-in voltage as a function of the quality factor, depending on the nonlinear factor $\beta$. Simulation is carried out to determine the exact pull-in voltage at which snapping is produced when a voltage step is applied. The values are normalized for better comparison, by dividing the calculated pull-in voltage by the SPV. For high- $Q$ environments, the pull-in voltage corresponds to the calculated DPV. For low- $Q$ environments, the pull-in voltage corresponds to the calculated SPV. The evolution is similar regardless of the nonlinear component.

and the corresponding DPV is

$$
\mathrm{DPV}=\sqrt{\frac{2 K g_{0}^{2}-16 K y_{\mathrm{uns}}^{2}+20 K y_{\mathrm{uns}} g_{0}+3 K_{3} y_{\mathrm{uns}}^{2} g_{0}^{2}}{32 C_{0}}} .
$$

Again, if a linear spring is used, the analytical expression has a simplified form. In this case, the voltage limit has always the unstable equilibrium at the center of the gap and is described by the following expression [32]:

$$
y_{\text {uns }}=\frac{g_{0}}{2} \quad \mathrm{DPV}=\sqrt{\frac{1}{4} \frac{K g_{0}^{2}}{C_{0}}} .
$$

As can be observed, the expressions have some similarities with the static case. Figs. 5 and 6 show the evolution of the static and dynamic pull-in parameters with the variation of the nonlinear factor $\beta$. Static and dynamic parameters behave in the same manner. Values of $\beta$ higher than 20 indicate the suitability of a linear model. Values of $\beta$ smaller than 2 indicate that the nonlinearities are predominant. In the case of the dynamic pull-in displacement, the maximum displacement during the evolution reaches up to $3 / 4$ of the gap in the case of a completely nonlinear spring.

Consequently, (19) and (20) expand prior DPV formulations to the whole range of values of nonlinear springs.

Another important aspect is the relationship between the SPV and DPV. Fig. 8 shows the analysis of the pull-in voltage as a function of the damping of the system $\zeta$ and the nonlinear factor $\beta$. The quality factor $Q=(M K)^{1 / 2} / B=1 / 2 \zeta$ is introduced as a usual parameter to evaluate the damping. As can be observed, in highly damped systems $(Q \approx 0)$, the voltage needed to produce snapping corresponds to the SPV. As the 


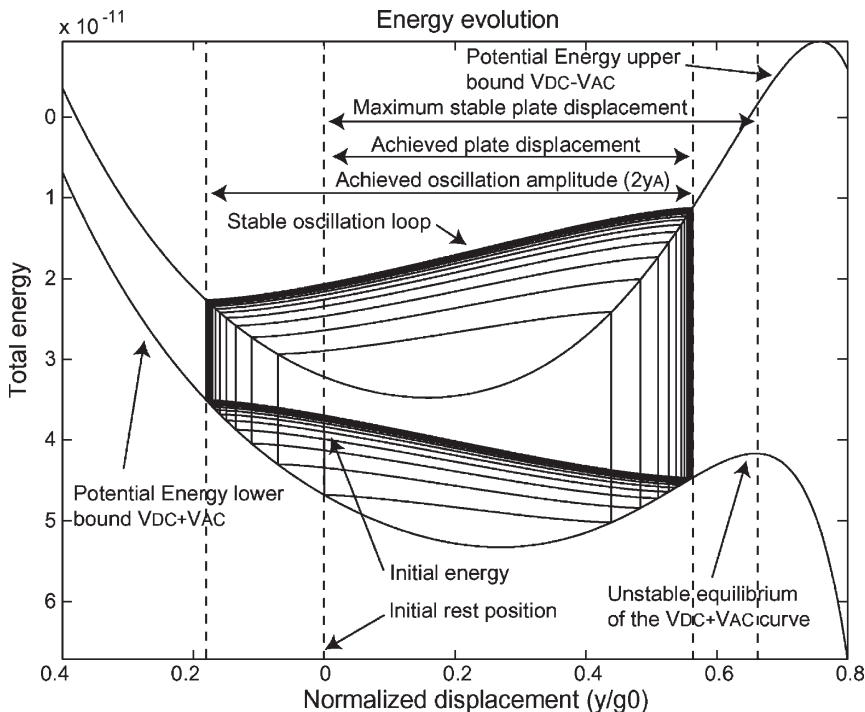

(a)

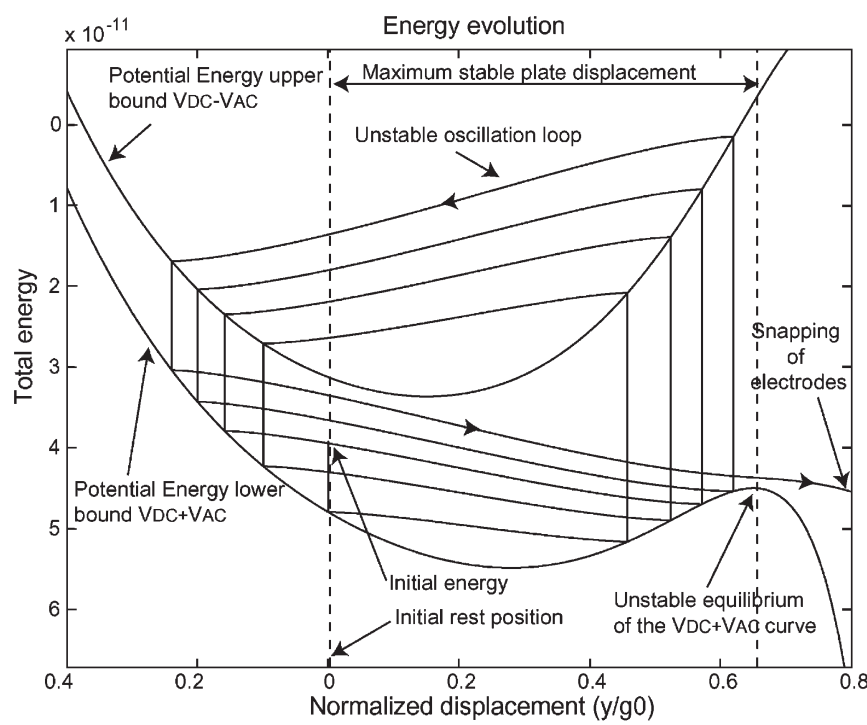

(b)

Fig. 9. Potential energy curves bound the system oscillation. (a) Stable oscillation is obtained with a $75-\mathrm{V}_{\mathrm{dc}}$ bias voltage and a $7-\mathrm{V}_{\mathrm{ac}}$ amplitude. (b) Oscillation is unstable with $75 \mathrm{~V}_{\mathrm{dc}}$ and $8 \mathrm{~V}_{\mathrm{ac}}$. Beginning from the static initial position, the amplitude of oscillation increases until it reaches the unstable equilibrium point at $V_{\mathrm{dc}}+V_{\mathrm{ac}}$, resulting in snapping.

quality factor increases, the voltage value decreases until it reaches the DPV. This happens for the whole range of spring values, from the linear case to the completely nonlinear case, and with the same pattern.

\section{Resonant Case}

In those cases where the system is dynamically actuated at resonance, as in oscillators, vibratory accelerometers, or gyroscopes, the stability analysis becomes more complex.

Under forced oscillation, the voltage varies with time, i.e., $V(t)=V_{\mathrm{dc}}+V_{\mathrm{ac}}(t)$, which means that the energy equilibrium points given by $d \mathbf{E} / d y$ are changing continuously. Thus,

$$
\frac{d \mathbf{E}}{d y}(t)=K \cdot y+K_{3} \cdot y^{3}-\frac{1}{2} \frac{C_{0}}{g_{0}\left(1-\frac{y}{g_{0}}\right)^{2}} V(t)^{2}=0 \text {. }
$$

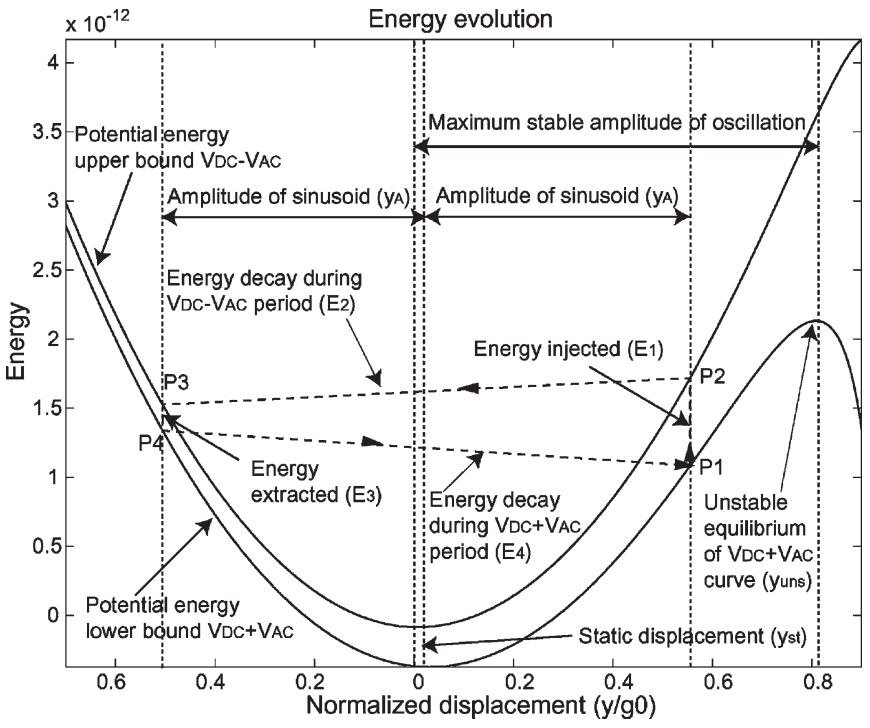

Fig. 10. Resonant pull-in algorithm. The energy evolution presented for the actuation voltage is stable as it generates a closed loop. The energy injected by the actuation is balanced with the damping losses.

As in the dynamic case, the potential energy curves bound the evolution of the total energy of the system. Consequently, their analysis allows to determine the maximum amplitude of oscillation that can be achieved without reaching the pull-in zone (Fig. 9). Equation (22) provides the condition for the extreme points of the energy function. Solving the equation for $V(t)=V_{D}+V_{\text {ac }}$, and discriminating maximum and minimum points using the second derivative, we can define $y_{\text {uns }}$ as the unstable equilibrium (maximum) of the $V_{D}+V_{\text {ac }}$ potential energy curve. Oscillations smaller than $y_{\text {uns }}$ are stable, whereas larger oscillations lead to pull-in [26].

In the resonant case, energy is continuously pumped into the system trying to reach the resonant frequency. Then, conceptually, stable actuation occurs while the energy of the system is confined in the valley of the potential energy.

If the alternating voltage $V_{\mathrm{ac}}(t)$ is considered to be a square function (without loss of generality, the analysis can be extended to other driving functions), at each half-period, the system behaves like in the dynamic case when a constant load is applied. When the voltage changes, the energy of the system jumps to the other energy region (Fig. 9).

As shown in Fig. 10, using the resonant pull-in algorithm [26], an oscillating loop in the energy domain close to the maximum amplitude can be generated to analyze the stability of the oscillation. For a complete driving voltage time period, an oscillating loop is constructed, estimating the energy decay from the value of the quality factor of the system. When the loop is closed, amplitude increase determines that the system is unstable, whereas amplitude decrease indicates that the system is stable.

\section{RESONANT PUll-In CONDITION}

The RPC can be derived using the energy evolution in a steady-state oscillation loop presented in the previous section.

The energy decay during the oscillation is controlled by the damping constant $B$. Assuming that the oscillation is 
sinusoidal, i.e., $y(t)=y_{A} \sin (\omega t)$, the value of the energy losses due to damping forces at each half-period can be estimated as

$$
\mathbf{E}_{\text {lost }}=-B y_{A}^{2} \omega \frac{\pi}{2}
$$

where $y_{A}$ is the amplitude of oscillation, and $\omega$ is the resonant frequency of oscillation of the system. The resonant frequency is usually different from the natural frequency of the mechanical system, i.e., $\omega_{n}=(K / M)^{1 / 2}$.

Consequently, the stability of oscillation will depend on the energy balance between the energy gained due to $V(t)=V_{\mathrm{dc}}+$ $V_{\text {ac }}(t)$ actuation and the energy lost due to damping [21].

In the energy oscillation loop, four energy terms are considered: $\mathbf{E}_{1}, \mathbf{E}_{2}, \mathbf{E}_{3}$, and $\mathbf{E}_{4}$ (Fig. 10). The initially gained energy $\mathbf{E}_{1}$, when moving from $V_{\mathrm{dc}}+V_{\mathrm{ac}}$ curve to $V_{\mathrm{dc}}-V_{\mathrm{ac}}$ curve, is

$$
\begin{aligned}
\mathbf{E}_{1}=[ & \frac{1}{2} K\left(y_{\mathrm{st}}+y_{A}\right)^{2}+\frac{1}{4} K_{3}\left(y_{\mathrm{st}}+y_{A}\right)^{4} \\
& \left.-\frac{1}{2} \frac{C_{0}\left(V_{\mathrm{dc}}-V_{\mathrm{ac}}\right)^{2}}{\left(1-\left(y_{\mathrm{st}}+y_{A}\right) / g_{0}\right)}\right] \\
- & {\left[\frac{1}{2} K\left(y_{\mathrm{st}}+y_{A}\right)^{2}+\frac{1}{4} K_{3}\left(y_{\mathrm{st}}+y_{A}\right)^{4}\right.} \\
& \left.\quad-\frac{1}{2} \frac{C_{0}\left(V_{\mathrm{dc}}+V_{\mathrm{ac}}\right)^{2}}{\left(1-\left(y_{\mathrm{st}}+y_{A}\right) / g_{0}\right)}\right] \\
= & \frac{2 C_{0} V_{\mathrm{dc}} V_{\mathrm{ac}}}{\left(1-\left(y_{\mathrm{st}}+y_{A}\right) / g_{0}\right)}
\end{aligned}
$$

where $y_{\mathrm{st}}$ is the position displacement of the electrode due to the $V_{\mathrm{dc}}$ bias. In this expression, $y_{\mathrm{st}}+y_{A}$ represents the effective maximum position in the gap.

The energy losses due to damping during the $V_{\mathrm{dc}}-V_{\mathrm{ac}}$ halfperiod $\left(\mathbf{E}_{2}\right)$ are

$$
\mathbf{E}_{2}=-B y_{A}^{2} \omega \frac{\pi}{2}
$$

The energy reduction when moving from $V_{\mathrm{dc}}-V_{\mathrm{ac}}$ curve to $V_{\mathrm{dc}}+V_{\mathrm{ac}}$ curve, which is obtained in a similar way as in (24), is

$$
\mathbf{E}_{3}=-\frac{2 C_{0} V_{\mathrm{dc}} V_{\mathrm{ac}}}{\left(1-\left(y_{\mathrm{st}}-y_{A}\right) / g_{0}\right)}
$$

where $y_{\mathrm{st}}-y_{A}$ represents the effective minimum amplitude position in the gap.

Finally, the energy losses due to damping during the $V_{\mathrm{dc}}+$ $V_{\mathrm{ac}}$ half-period $\left(E_{4}\right)$ are

$$
\mathbf{E}_{4}=-B y_{A}^{2} \omega \frac{\pi}{2}
$$

If the system has been actuated at a stable resonant frequency, there must exist an amplitude of oscillation where the energy balance of the loop is zero. Consequently, the following equation has to be satisfied:

$$
\mathbf{E}_{1}+\mathbf{E}_{2}+\mathbf{E}_{3}+\mathbf{E}_{4}=\frac{4 C_{0} g_{0} V_{\mathrm{dc}} V_{\mathrm{ac}} y_{A}}{\left(g_{0}-y_{\mathrm{st}}\right)^{2}-y_{A}^{2}}-B y_{A}^{2} \omega \pi=0 .
$$

Rearranging terms in (28), the amplitude of oscillation $y_{A}$ of the stable loop can be obtained from the following equation:

$$
y_{A}^{3}-\left(g_{0}-y_{\mathrm{st}}\right)^{2} y_{A}+\frac{4 C_{0} g_{0} V_{\mathrm{dc}} V_{\mathrm{ac}}}{B \omega \pi}=0 .
$$

The equation can be solved analytically. However, to predict the existence of stable oscillation, we only need to know the type of solutions of (29). This analysis can be done through the third-order polynomial discriminant $D$

$$
D=-\frac{1}{27}\left(g_{0}-y_{\mathrm{st}}\right)^{6}+\frac{4 C_{0}^{2} g_{0}^{2} V_{\mathrm{dc}}^{2} V_{\mathrm{ac}}^{2}}{B^{2} \omega^{2} \pi^{2}} .
$$

In a cubic polynomial, $D=0$ identifies the transition between all-real solutions and the existence of complex solutions. Applied to the parallel-plate system, this equation leads to

$$
\mathrm{RPC}=V_{\mathrm{dc}} V_{\mathrm{ac}}=\frac{B \omega \pi\left(g_{0}-y_{\mathrm{st}}\right)^{3}}{6 \sqrt{3} C_{0} g_{0}}
$$

which provides the maximum value of the product $V_{\mathrm{dc}} V_{\mathrm{ac}}$ producing stable oscillation.

Once the $V_{\mathrm{dc}}$ load applied to the system is defined, the static displacement $y_{\text {st }}$ can be calculated, and accordingly, the real resonant frequency $\omega$ can be estimated, for example, using the voltage-corrected frequency

$$
\omega_{e}=\sqrt{\frac{K-\frac{C_{0} V_{\mathrm{dc}}^{2}}{g_{0}^{2}}}{M}} .
$$

Consequently, the RPC defines a constructive domain of $V_{\mathrm{dc}}$ and $V_{\text {ac }}$ actuation voltages versus quality factor, preserving the stability of the parallel-plate actuation.

As can be observed, the introduction of the nonlinear spring in the model does not change the RPC, which is equal to that derived in the linear spring case [27].

In the case of only $V_{\mathrm{ac}}$ actuation, two-sided push-pull actuation is needed with square-function voltages, and (31) transforms to

$$
\mathrm{RPC}=V_{\mathrm{ac}}=\sqrt{\frac{B \omega \pi g_{0}^{2}}{\sqrt{27} C_{0}}}
$$

as presented in [21]. Again, the derivation holds even considering large amplitudes and nonlinear spring behavior.

It is important to notice that at resonant frequency, the maximum amplitude of oscillation is limited. In [21], it was indicated that in two-sided actuation, the maximum amplitude of oscillation is

$$
y_{A}=\frac{g_{0}}{\sqrt{3}}
$$

which corresponds to the maximum displacement in the gap. 
TABLE I

Structural PARAMETERS OF THE FABRicATED DEVICES

\begin{tabular}{|l|c|c|}
\hline & 1500-Model & 2500-Model \\
\hline \hline Stiffness K & $2.066 \mathrm{~N} / \mathrm{m}$ & $1.766 \mathrm{~N} / \mathrm{m}$ \\
\hline Nonlinear Stiffness $\mathrm{K}_{3}$ & $4.678 \cdot 10^{10} \mathrm{~N} / \mathrm{m}^{3}$ & $4.463 \cdot 10^{10} \mathrm{~N} / \mathrm{m}^{3}$ \\
\hline Mass M & $6.753 \cdot 10^{-10} \mathrm{Kg}$ & $3.830 \cdot 10^{-9} \mathrm{Kg}$ \\
\hline Initial gap g & $11.4 \mu \mathrm{m}$ & $11.3 \mu \mathrm{m}$ \\
\hline Parallel-plate actuator $\mathrm{A}_{c}$ & $800 \cdot 15 \mu \mathrm{m}^{2}$ & $800 \cdot 75 \mu \mathrm{m}^{2}$ \\
\hline Beam length L & $1500 \mu$ & $2500 \mu$ \\
\hline Beam width h & $5.6 \mu$ & $5.3 \mu$ \\
\hline Device thickness b & $15 \mu$ & $70 \mu$ \\
\hline Nominal frequency $\mathrm{f}_{n}$ & $8.804 \mathrm{kHz}$ & $3.41 \mathrm{kHz}$ \\
\hline$\beta$ coefficient & 0.34 & 0.31 \\
\hline \hline
\end{tabular}

In the case of $V_{\mathrm{dc}}+V_{\mathrm{ac}}$ actuation, this limitation translates to

$$
y_{A}=\frac{g_{0}-y_{\mathrm{st}}}{\sqrt{3}}
$$

which is obtained by substitution of the RPC in (29). Consequently, the maximum displacement in the capacitive gap is

$$
y_{\max }=y_{\mathrm{st}}+\frac{g_{0}-y_{\mathrm{st}}}{\sqrt{3}} .
$$

\section{EXPERIMENTAL RESULTS}

A family of silicon-on-insulator (SOI) resonators was fabricated (Fig. 1) to experimentally validate the RPC. The structures were fabricated in the Centro Nacional de Microelectrònica, Barcelona, using a one-mask bulk-micromachining process that is based on deep reactive-ion etching through the 15- or 70- $\mu \mathrm{m}$ device layer of SOI wafers [33]. In Table I, the main parameters of two of the resonators used for experiments are summarized. The parameters have been obtained from the initial fabrication designs and corrected taking into account the observed fabrication imperfections.

In the case of the 1500-Model (Table I), the classical pullin analysis defines that the static instability (18) occurs at $75.61 \mathrm{~V}$, when the gap becomes approximately $7.6 \mu \mathrm{m}$. Introduction of the existing nonlinear effects allows to conclude that the allowed driving voltage (17) is in fact larger, i.e., $91.69 \mathrm{~V}$, and the final remaining gap is much smaller, i.e., $6.01 \mu \mathrm{m}$ (16). No equilibrium points exist at smaller gap for voltages higher than the SPV, as can be observed in Fig. 4.

The analysis can be extended introducing an actuation voltage as a nonsmooth function of time, as it would be if the voltage were applied as a step function. In this case, the dynamics of the system play their role in defining the maximum driving voltages. Again, the classical DPV formulation (21) gives $69.45 \mathrm{~V}$ as the minimum voltage that can produce dynamic snapping. In this paper, it has been shown that the introduction of the nonlinear spring constant has its effect on the DPV. Using (20), it can be observed that the DPV increases up to $84.41 \mathrm{~V}$ due to the nonlinear forces. In this case, the minimum gap during the evolution would be $4.15 \mu \mathrm{m}$ from (19). The snapping for voltages higher than this value will depend on the damping of the system, which is directly
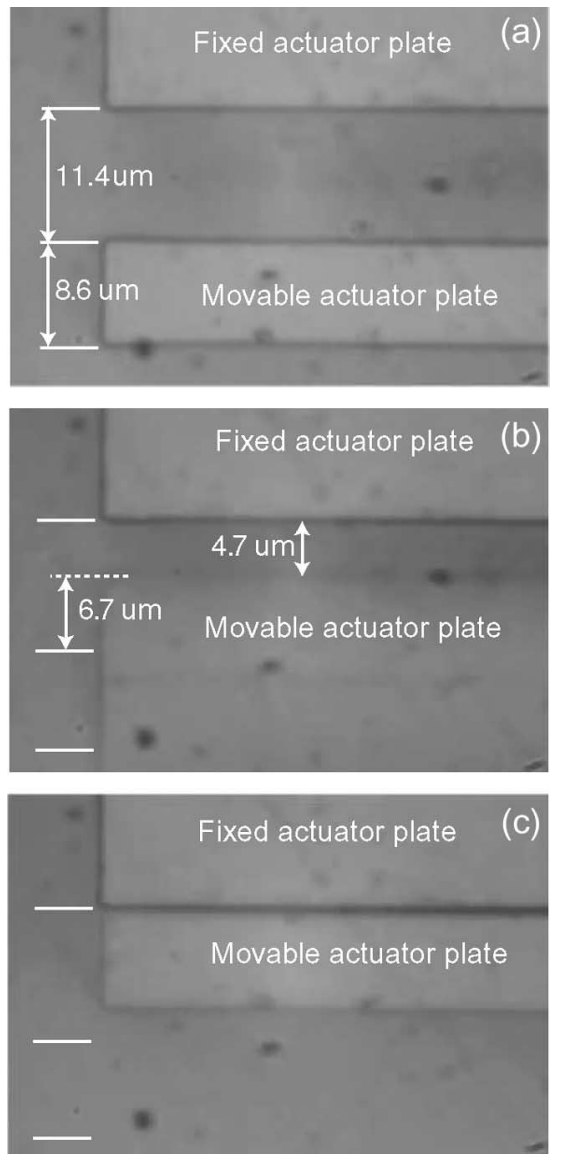

Fig. 11. Set of pictures presenting evolution of the amplitude of oscillation of the resonator in Fig. 1 due to changes in ac-dc driving voltages; The pictures show a close-up of the parallel-plate electrodes. The SPV is $91.69 \mathrm{~V}$, and the DPV is $84.41 \mathrm{~V}$. (a) Beam at rest. (b) Beam oscillating with $75 \mathrm{~V}_{\mathrm{dc}}$ and $6.8 \mathrm{~V}_{\mathrm{ac}}$. (c) Beam snapped after applying a combination of $75-\mathrm{V}_{\mathrm{dc}}$ and 6.9- $\mathrm{V}_{\mathrm{ac}}$ drive voltages.

proportional to the air pressure of packaged microdevices. For low-pressure or vacuum conditions, voltages higher than DPV would imply snapping (Fig. 8).

As can be observed, calculation of SPV and DPV are very much dependent on the nonlinearities of the system (Figs. 5 and 6). In the resonator considered in this example, the $\beta$ factor is 0.34 , which translates to the increases of the needed voltage by $20 \%$. This conclusion is important as it extends the stable range for nonsnapping applications. On the contrary, it is a drawback for applications where pull-in is desired, showing that higher voltages are needed than those classically predicted.

These results show the importance of dynamics and nonlinearities when studying the stability of MEMS devices. They play an important role when the structure is dynamically actuated to its resonant frequency. In resonant devices, the instability (or snapping) occurs at much smaller voltages. In the example presented in Fig. 11, snapping occurred with a $75-\mathrm{V}_{\mathrm{dc}}$ bias and a $6.9-\mathrm{V}_{\mathrm{ac}}$ peak amplitude. (The sum of the voltages is smaller than DPV $=84.41 \mathrm{~V}$.) During oscillation, large stable amplitudes have been reached, or equivalently smaller gaps, approximately $4.7 \mu \mathrm{m}$ in our case (60\% gap reduction). As can be observed, significantly larger amplitude of actuation can be achieved when dynamic actuation is used. The "overshoot" 


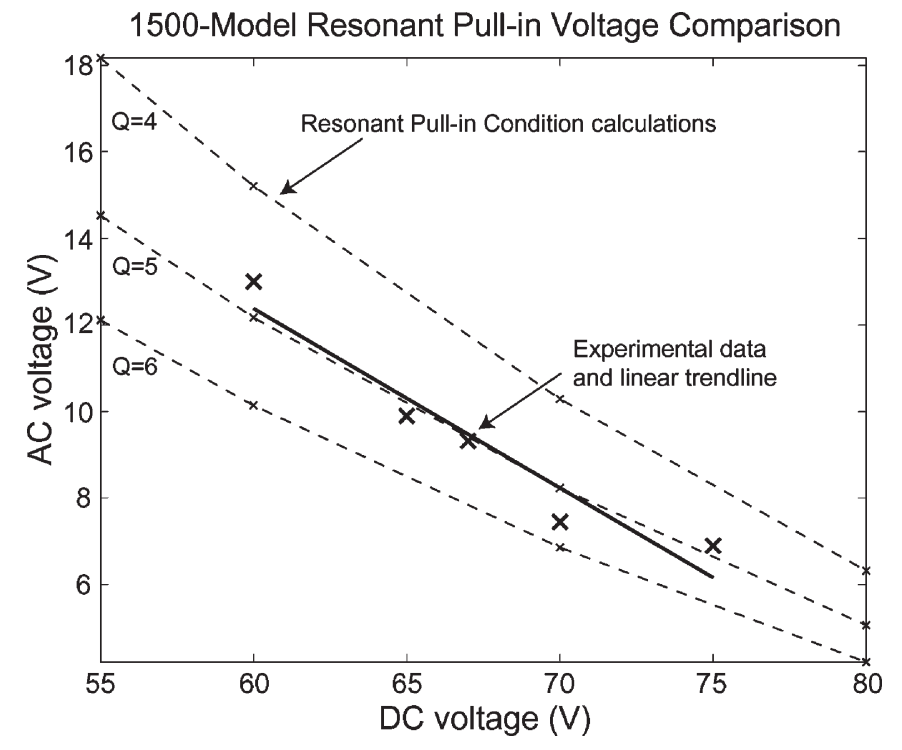

Fig. 12. Maximum combinations of $V_{\mathrm{dc}}$ and $V_{\mathrm{ac}}$ voltages for the different values of the quality factor $Q$ for the 1500-Model. Values estimated with the RPC are presented with the experimental data in air. The trend of experimental values agrees with predictions.

effect of the static equilibrium is explained by the gained kinetic energy of the system, which allows it to return to the stable region of actuation.

This dynamic behavior can be predicted using the RPC. With resonant devices, it plays the same role as the SPV in positioning applications or the DPV in switching applications.

The experimental snapping values obtained for the 1500-Model in air are presented in Fig. 12. In the same plot, calculations of the RPC are used to produce the combination of maximum allowed $V_{\mathrm{dc}}$ and $V_{\mathrm{ac}}$ voltages for values of the quality factor ranging from 4 to 6 , which correspond to the range of $Q$ of the device in air. As can be observed, experimental data are consistent with the analytically derived regions of instability.

Furthermore, the same experimental testing was done for the 2500-Model (Fig. 13). In this comparison, results of resonant pull-in voltage that were obtained via direct time integration of the system equations (14) at the testing environment conditions $(Q=2$ for the 2500-Model) are also provided. As can be observed, RPC predictions show good agreement with experimental data.

The results in Fig. 13 also show that RPC predictions are close to the values obtained via numerical time integration of the system equations (14). This is important because within the RPC calculation, the resonant frequency is approximated by the voltage-corrected frequency (32). Fig. 13 illustrates that this approximation has a small effect (5\% error) in predicting the snapping values.

\section{CONCLUSION}

Operation of electrostatically actuated MEMS with amplitudes much higher than $1 / 3$ of the initial actuation gap can be achieved with appropriate selection of actuation voltages. The kinetic energy of the system gained during actuation allows the
2500-Model Resonant Pull-in Voltage Comparison

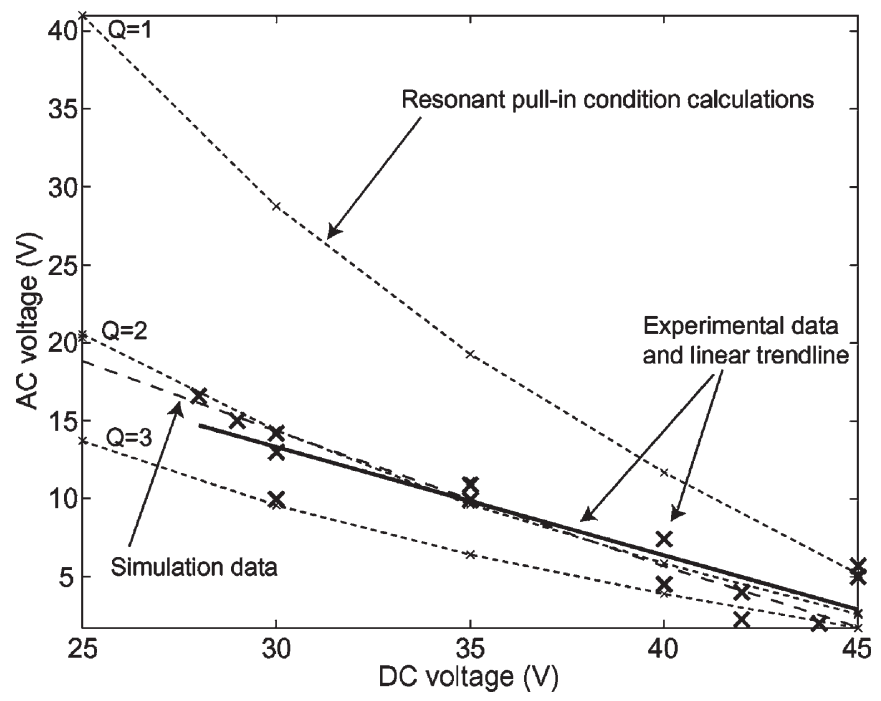

Fig. 13. Maximum combinations of $V_{\mathrm{dc}}$ and $V_{\mathrm{ac}}$ voltages for the different values of the quality factor $Q$ for the 2500-Model. RPC values are presented together with experimental data obtained in air and data from the numerical time integration of system equations for $Q=2$. The three curves show good agreement.

system to travel beyond the static equilibrium, reaching large amplitudes of oscillation without snapping.

Energy analysis has been used to derive the RPC, which provides the combination of maximum $V_{\mathrm{dc}}$ and $V_{\mathrm{ac}}$ voltages that can be used to actuate the system without producing snapping at resonance frequency.

RPC has been shown to predict snapping in fabricated MEMS devices actuated at resonance. However, it is known that transient response can affect the nonlinear dynamic behavior of the system and lead the system to pull-in at lower voltages [22].

RPC can be a useful tool to design dynamic MEMS, along with the estimation of the SPV and the DPV. The derived stability limits represent upper limits to the dynamic pull-in, beyond which no stable motion can exist.

RPC can also be used as the first-order solution for iterative numerical simulation analysis or for prediction of the voltages needed for resonant switching applications.

It has also been shown that the RPC can deal with nonlinear spring models, expanding the previously reported formulations.

\section{REFERENCES}

[1] G. Kovacs, Micromachined Transducers Sourcebook. New York: McGraw-Hill, 1998.

[2] E. S. Hung and S. D. Senturia, "Extending the travel range of analogtuned electrostatic actuators," J. Microelectromech. Syst., vol. 8, no. 4, pp. 497-505, Dec. 1999.

[3] M. A. Rosa, D. D. Bruyker, A. R. Völkel, E. Peeters, and J. Dunec, "A novel external electrode configuration for the electrostatic actuation of MEMS based devices," J. Micromech. Microeng., vol. 14, no. 4, pp. 446-451, Apr. 2004.

[4] H. Busta, R. Amantea, D. Furst, J. M. Chen, M. Turowski, and C. Mueller, "A MEMS shield structure for controlling pull-in forces and obtaining increased pull-in voltages," J. Micromech. Microeng., vol. 11, no. 6, pp. 720-725, 2001.

[5] P. Chu and S. Pister, "Analysis of closed-loop control of parallel-plate electrostatic microgrippers," in Proc. IEEE Int. Conf. Robot. and Autom., 1994, vol. 1, pp. 820-825. 
[6] J. Seeger and S. Crary, "Stabilization of electrostatically actuated mechanical devices," in Proc. Int. Conf. Solid State Sens. and Actuators, TRANSDUCERS, 1997, vol. 2, pp. 1133-1136.

[7] L. Castañer, J. Pons, R. Nadal-Guardia, and A. Rodríguez, "Analysis of extended actuation range of electrostatic actuators by current pulse drive," Sens. Actuators A, Phys., vol. 90, no. 3, pp. 181-190, 2001.

[8] J. Seeger and B. Boser, "Charge control of parallel-plate, electrostatic actuators and the tip-in instability," J. Microelectromech. Syst., vol. 12, no. 5, pp. 656-671, Oct. 2003.

[9] R. K. Gupta and S. Senturia, "Pull-in time dynamics as a measure of absolute pressure," in Proc. 10th Аnпи. Int. Workshop MEMS, 1997, pp. 290-294.

[10] L. Rocha, E. Cretu, and R. Wolffenbuttel, "Pull-in dynamics: Analysis and modeling of the transitional regime," in Proc. 17th IEEE Int. Conf. MEMS, 2004, pp. 249-252.

[11] W. C. Xie, H. P. Lee, and S. P. Lim, "Nonlinear dynamic analysis of MEMS switches by nonlinear modal analysis," Nonlinear Dyn., vol. 31, no. 3, pp. 243-256, Feb. 2003.

[12] E. Saucedo-Flores, R. Ruelas, M. Flores, C. Ying, and C. Jung-chih, "Dynamic behavior modeling of MEMS parallel plate capacitors," in Proc. IEEE PLANS, 2004, pp. 15-19.

[13] S. Krylov and R. Maimon, "Pull-in dynamics of an elastic beam actuated by continuously distributed electrostatic force," Trans. ASME, J. Vib. Acoust. Stress Reliab. Des., vol. 126, no. 3, pp. 332-342, Jul. 2004.

[14] M. I. Younis and A. H. Nayfeh, "A study of the nonlinear response of a resonant microbeam to an electric actuation," Nonlinear Dyn., vol. 31, no. 1, pp. 91-117, Jan. 2003.

[15] S. K. De and N. Aluru, "Complex nonlinear oscillations in electrostatically actuated microstructures," J. Microelectromech. Syst., vol. 15, no. 2, pp. 355-369, Apr. 2006.

[16] M. Napoli, R. Turner, and B. K. Bamieh, "Understanding mechanical domain parametric resonance in microcantilevers," in Proc. IEEE 16th Аnnu. Int. Conf. MEMS, Kyoto, Japan, 2003, pp. 169-172.

[17] S. Krylov, I. Harari, and Y. Cohen, "Stabilization of electrostatically actuated microstructures using parametric excitation," J. Micromech. Microeng., vol. 15, no. 6, pp. 1188-1204, Jun. 2005.

[18] S. Beeby, G. Ensell, B. Baker, M. Tudor, and N. White, "Micromachined silicon resonant strain gauges fabricated using SOI wafer technology," J. Microelectromech. Syst., vol. 9, no. 1, pp. 104-111, Mar. 2000

[19] S. Sung, J. Lee, T. Kang, and J. W. Song, "Development of a tunable resonant accelerometer with self-sustained oscillation loop," in Proc. IEEE Nat. Aerosp. and Electron. Conf., 2000, pp. 354-361.

[20] C. C. Painter and A. M. Shkel, "Active structural error suppression in MEMS vibratory rate integrating gyroscopes," IEEE Sensors J., vol. 3, no. 5, pp. 595-606, Oct. 2003.

[21] J. I. Seeger and B. E. Boser, "Parallel-plate driven oscillations and resonant pull-in," in Proc. Solid-State Sensor, Actuator and Microsyst. Workshop, Hilton Head Island, SC, 2002, pp. 313-316.

[22] A. H. Nayfeh, M. I. Younis, and E. M. Abdel-Rahman, "Dynamic analysis of MEMS resonators under primary-resonance excitation," in Proc. IDETC/CIE ASME, 2005, pp. 397-404.

[23] A. H. Nayfeh and M. I. Younis, "Dynamics of MEMS resonators under superharmonic and subharmonic excitations," J. Micromech. Microeng., vol. 15 , no. 10, pp. 1840-1847, Oct. 2005.

[24] A. H. Nayfeh, M. I. Younis, and E. M. Abdel-Rahman, "Dynamic pullin phenomenon in MEMS resonators," Nonlinear Dyn., vol. 48, no. 1/2, pp. 153-163, Apr. 2007.

[25] S. Lenci and G. Rega, "Control of pull-in dynamics in a nonlinear thermoelastic electrically actuated microbeam," J. Micromech. Microeng., vol. 16, no. 2, pp. 390-401, Feb. 2006.

[26] A. Fargas-Marques and A. M. Shkel, "On electrostatic actuation beyond snapping condition," in Proc. Eurosensors XIX, Barcelona, Spain, Sep. 2005, p. MB13.

[27] A. Fargas-Marques and A. M. Shkel, "On electrostatic actuation beyond snapping condition," in Proc. IEEE SENSORS, Irvine, CA, Nov. 2005, pp. 600-603.

[28] Y. Nemirovsky and O. Bochobza-Degani, "A methodology and model for the pull-in parameters of electrostatic actuators," J. Microelectromech. Syst., vol. 10, no. 4, pp. 601-615, Dec. 2001.

[29] T. A. W. Roessig, "Integrated MEMS tuning fork oscillators for sensor applications," Ph.D. dissertation, Univ. California, Berkeley, CA, 1998.

[30] J. Huang, K. Liew, C. Wong, S. Rajendran, M. Tan, and A. Q. Liu, "Mechanical design and optimization of capacitive micromachined switch," Sens. Actuators A, Phys., vol. 93, no. 3, pp. 273-285, Oct. 2001.

[31] M. Younis, E. M. Abdel-Rahman, and A. Nayfeh, "A reduced-order model for electrically actuated microbeam-based MEMS," J. Microelectromech. Syst., vol. 12, no. 5, pp. 672-680, Oct. 2003.
[32] R. K. Gupta, E. Hung, Y. Yang, G. Ananthasuresh, and S. Senturia, "Pullin dynamics of electrostatically-actuated beams," in Proc. Solid-State Sens. and Actuators Workshop, Late News Session, 1996, pp. 1-2.

[33] C. Acar and A. M. Shkel, "Inherently robust micromachined gyroscopes with 2-DOF sense-mode oscillator," J. Microelectromech. Syst., vol. 15, no. 2, pp. 380-387, Apr. 2006.

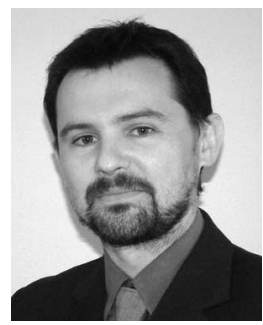

Andreu Fargas-Marques received the Diploma (with excellence) in industrial engineering from the Technical University of Catalonia (UPC), Barcelona, Spain, in 2000, and the M.S. degree in mechanical and aerospace engineering from the University of California, Irvine, in 2001. He is currently working toward the Ph.D. degree in modeling and control of parallel-plate electrostatically actuated MEMS at the Institute of Industrial and Control Engineering, UPC.

His research interests are the control of MEMS, static forces such as resonators or switches. particularly those actuated via parallel-plate electro-

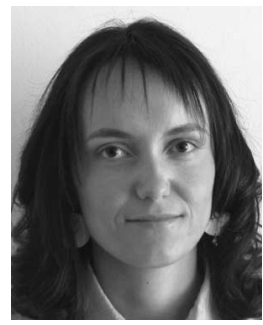

Jasmina Casals-Terré received the M.S. degree in mechanical engineering from the Technical University of Catalonia (UPC), Terrassa, Spain, in 2000, and the M.S. degree in mechanical and aerospace engineering from the University of California, Irvine, in 2002. She is currently working toward the Ph.D. degree in design, fabrication, and testing of bistable microswitches in the Department of Mechanical Engineering, UPC.

Her research interests are in bistable MEMS, such as switches or microvalves.

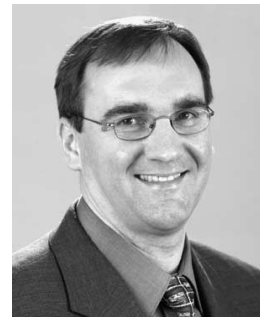

Andrei M. Shkel (S'95-A'98) received the Diploma (with excellence) in mechanics and mathematics from Moscow State University, Moscow, Russia, in 1991, and the Ph.D. degree in mechanical engineering from the University of Wisconsin, Madison, in 1997.

$\mathrm{He}$ has been a member of the Faculty of the University of California, Irvine (UCI), since 2000 , where he is an Associate Professor in the Department of Mechanical and Aerospace Engineering, Department of Electrical Engineering and Computer Sciences, and Department of Biomedical Engineering. He is also the Director of the UCI Microsystems Laboratory. After receiving the Ph.D. degree, he joined the Berkeley Sensor and Actuator Center as a Postdoctoral Researcher. He then held research and consulting positions in several high-tech and venture companies. He is the holder of eight U.S. and international patents (with 13 pending) on micromachined angle-measuring gyroscopes, wide-bandwidth rate gyroscopes, design and fabrication of light manipulators and tunable optical filters, and hybrid micromachining processes. His professional interests, which are reflected in more than 100 publications, include solid-state sensors and actuators, MEMS-based neuroprosthetics, sensor-based intelligence, and control theory.

Dr. Shkel is a member of the American Society of Mechanical Engineers (ASME), the Institute of Navigation, and the International Society for Optical Engineers (SPIE). He was a Guest Editor for two special issues of the IEEE SENSORS Journal, the General Chair of the 2005 IEEE Sensors Conference, and the Vice General Chair and Publications Chair of the 2002, 2003, 2004, and 2006 IEEE Sensors Conferences. He was the recipient of the $2006 \mathrm{Re}-$ search Award of the UCI School of Engineering, the 2005 National Science Foundation CAREER Award, the 2002 George E. Brown, Jr., Award, and a 2001 Fellowship from the Japanese Advanced Science Institute. 